\title{
SPECTRAL ANALYSIS OF RANDOM SPARSE MATRICES
}

\author{
TOMONORI ANDO, YOSHIYUKI KABASHIMA, HISANAO TAKAHASHI, OSAMU \\ WATANABE, MASAKI YAMAMOTO
}

\begin{abstract}
We study $n \times n$ random symmetric matrices whose entries above the diagonal are iid random variables each of which takes 1 with probability $p$ and 0 with probability $1-p$, for a given density parameter $p=\alpha / n$ for sufficiently large $\alpha$. For a given such matrix $A$, we consider a matrix $A^{\prime}$ that is obtained by removing some rows and corresponding columns with too many value 1 entries. Then for this $A^{\prime}$, we show that the largest eigenvalue is asymptotically close to $\alpha+1$ and its eigenvector is almost parallel to all one vector $(1, \ldots, 1)$.
\end{abstract}

\section{INTRODUCTION}

We study the largest eigenvalues and their eigenvectors of random symmetric and sparse 0,1-matrices. Spectral analysis (of matrices) is in general to analyze eigenvalues and eigenvectors (of given matrices). Random matrices and their spectral analysis play key roles in many problems in information sciences. In particular, analyzing random symmetric 0,1-matrices has been shown quite important for the design and analysis of combinatorial algorithms such as graphs, etc; see, for example, a survey [1] of Alon for relation to graph algorithms, and a paper [3] for relation to SAT algorithms. Because of its importance, several detail spectral investigations have been made for random symmetric 0,1-matrices. Yet our understanding is still limited; many important questions have been left open, and there seem to exist some interesting but unknown spectral properties. In fact, using powerful approximation methods developed in statistical physics, many interesting and useful spectral properties have been shown approximately under certain heuristic assumptions. We have been trying to give rigorous justifications to such approximate analysis, and this paper reports one set of results on the largest eigenvalue and its eigenvector of a random symmetric and sparse 0, 1-matrix.

For explaining previous related work, we first introduce some notions and notations for discussing the results precisely. Let $\mathbf{N}$ denote the set of positive integers.

A part of this work has been done while the second, third, and fourth authors visited at Centre de Recerca de Matemàtica, Barcelona, Spain. This work is supported in part by Grantsin-Aid for Scientific Research on the Priority Areas "Deepening and Expansion of Statistical Mechanical Informatics" (22300003) and the JSPS Global COE program, "Computationism as a Foundation for the Sciences." 
Throughout this paper, we will use $n \in \mathbf{N}$ as a size parameter, a parameter determining the size of matrices and vectors. Let $[n]$ denote the set $\{1, \ldots, n\}$. Our analysis is stated asymptotically; that is, we consider the situation where $n$ is sufficiently large. In particular, by "w.h.p." we mean that a given event holds with probability approaching to 1 when $n \rightarrow \infty$.

Our target object is an $n \times n$ random symmetric matrix $A=\left(a_{i j}\right)$ whose diagonal entries are zeros and whose entries above the diagonal are iid random variables, each taking value 1 with probability $p$ and value 0 with probability $1-p$. A parameter $p$ is called a density parameter. In this paper, we consider "sparse" matrices, where $p$ is small and $A$ has on average not so many entries with value 1. Precisely, we consider the case where the density parameter is defined by $p=\alpha / n$ for some sufficiently large $\alpha>0$.

The largest eigenvalue and its eigenvector are the most important subjects of the spectral analysis. In this paper, we use $\lambda_{1}$ and $\chi_{1}$ to denote the largest eigenvalue of $A$ and the normalized eigenvector of this eigenvalue. Roughly speaking, we may expect that $\lambda_{1}$ is close to $p n$ and that $\chi_{1}$ is almost parallel to vector $\overline{\mathbf{1}}=(1, \ldots, 1) / \sqrt{n}$, or more quantitatively $\left|\left\langle\chi_{1}, \overline{\mathbf{1}}\right\rangle\right|$ is close to 1 . (Throughout this paper, we will use $a$ to denote the quantity $\left|\left\langle\chi_{1}, \overline{\mathbf{1}}\right\rangle\right|$.) To see these fundamental spectral properties, let us consider a matrix $\widehat{A}$ whose each entry takes the expectation of the corresponding entry of $A$; that is, $\widehat{A}$ 's each diagonal entry is 0 , and its each nondiagonal entry is $p$. For this $\widehat{A}$, it is easy to see that $\widehat{A} \mathbf{1}=p(n-1) \mathbf{1}$. That is, $p(n-1) \approx p n$ is one of the eigenvalues of $\widehat{A}$ and its eigenvector is parallel to 1 ; furthermore, it is not so hard to show that $p(n-1)$ is the largest eigenvalue. Thus, it seems that $\lambda_{1} \approx p n$ and $a \approx 1$ on average. In fact, results supporting this intuition have been shown in the literature, and they have been used as key facts for the spectral analysis of algorithms. The detail analysis, however, has not been done completely, in particular for the sparse case that we will study in this paper. Note that these properties may not hold in the sparse case. One reason is, as pointed out in the literature (e.g., [2]), the fact that the possibility to have some rows (and columns by symmetry) with unusually many value 1 entries is not so small; such rows and columns cause certain irregularity, which affects $\lambda_{1}$ and $\chi_{1}$. But even in this case, we can still expect similar properties if we remove such rows and columns.

Recently, by using powerful approximation methods developed in statistical physics, various spectral properties of random sparse matrices have been demonstrated [7]. Although their analysis uses certain approximations and heuristic hypotheses, the results that match very well to computer experiments seem quite informative and useful. For the above explained fundamental properties, they showed that if $\alpha>1$, then we have

$$
\lambda_{1} \approx \alpha+1, \text { and } a \approx \sqrt{1-\frac{1}{\alpha}}
$$


for random symmetric 0,1 -matrices created with density parameter $p=\alpha / n$ (and then modified by removing rows and columns with too many 1 entries). In this paper, we prove that these relations asymptotically hold w.h.p. for sufficiently large $\alpha$.

Note that $\alpha=p n$. Thus, $\lambda_{1} \approx \alpha+1=p n+1$ is different from our intuition $\lambda_{1} \approx p n$ by +1 . One can derive a similar estimate with the +1 term from the earlier result of [6] for the dense case where $p>\Omega(1)$. More recently, Krivelevich and Sudakov [8] analyzed the largest eigenvalue for the sparse case. While their result include some other case, the relation that we can show from their result is $\lambda_{1}=(1+o(1)) p n$, which is still not sharp enough to show this +1 term. Our result is the first one that gives a sharper estimate deriving the +1 term.

\section{Preliminaries and Key Lemma}

As mentioned in Introduction, we consider an $n \times n$ random symmetric matrix $A$ whose diagonal entries are zeros and whose entries above the diagonal are iid random variables, each taking value 1 with probability $p$ and value 0 with probability $1-p$. We will use symbols $\boldsymbol{\xi}, \boldsymbol{\eta}, \ldots$ to denote unit vectors. On the other hand, for general vectors, e.g., $\overline{\boldsymbol{v}}$, by $\overline{\boldsymbol{v}}$, we denote a unit vector $\boldsymbol{v} /\|\boldsymbol{v}\|$ that is obtained from $\boldsymbol{v}$ by normalization. Let $\mathbf{1}$ and $\overline{\mathbf{1}}$ denote all one vector $(1,1, \ldots, 1)$ and its normalization respectively. For each $i, 1 \leq i \leq n$, let $\chi_{i}$ denote the $i \mathrm{Th}$ largest eigenvector of $A$.

We rely on the analysis of random 0,1-matrices given by Alon and Kahale [2], which is based on the approach given in [5]. More specifically, we base the following lemma given by Coja-Oghlan et al. in [3, Lemma 45]. (Below, we use, e.g., \#S to denote the number of elements in the set $S$.)

Lemma 1. Let $A$ be a random matrix as explained above with density parameter $p=\alpha / n$. For some sufficiently large constant $d_{0}$, if $\alpha \geq d_{0}$, then for any constant $c_{1}>1$, there exists some $c_{2}>0$ such that the following three statements hold w.h.p. for the random matrix $A$.

(1) Let $V^{\prime \prime}=\left\{i \in\{1, \ldots, n\} \mid \sum_{j=1}^{n} a_{i j}>c_{1} p n\right\}$, and $V^{\prime}=\{1, \ldots, n\}-V^{\prime \prime}$. Let $n^{\prime}=\# V^{\prime}$ and $A^{\prime}=\left(a_{i j}\right)_{i, j \in V^{\prime}}$ be the induced $n^{\prime} \times n^{\prime}$ matrix. Then we have $n^{\prime} \geq\left(1-\exp \left(-p n / c_{2}\right)\right) n$.

(2) For any unit vector $\boldsymbol{\xi}$ such that $\boldsymbol{\xi} \perp \mathbf{1}$, we have $\left\|A^{\prime} \boldsymbol{\xi}\right\| \leq c_{2} \sqrt{p n^{\prime}}$.

(3) $\left\|A^{\prime} \overline{\mathbf{1}}-p n^{\prime} \overline{\mathbf{1}}\right\| \leq c_{2} \sqrt{p n^{\prime}}$.

The lemma states first that the number of rows (resp., columns) with unusually many 1 entries is not so large w.h.p. (for sufficiently large $\alpha$ ). It then states that after removing such rows and columns the remaining matrix, i.e., the matrix $A^{\prime}$ of the lemma, behaves w.h.p. more or less similar to the "average" matrix. This $A^{\prime}$ will be the target of our analysis of this paper. (In the next section, we will use $A$ to denote this $A^{\prime}$.) 
We prepare some more technical lemmas. Here we introduce our order notations that will be used throughout this paper. For any positive function $f(n)$, we use $O(f(n))$ and $o(f(n))$ to denote some positive functions $f_{1}(n)$ and $f_{2}(n)$ that are defined in each context to satisfy $\exists c_{1}>0, \exists n_{1} \in \mathbf{N}, \forall n \geq n_{1}\left[f_{1}(n) \leq c_{1} f(n)\right]$ and $\forall c>0, \exists n_{2} \in \mathbf{N}, \forall n \geq n_{2}\left[f_{2}(n) \leq c f(n)\right]$ respectively. Note that we consider only positive functions, which is different from the standard order notations. This is for simplifying expressions and derivations. For example, for our notations, it does make sense to write expressions such as $f(n)<o(1)$ or use derivations such as $(\sqrt{p n}+o(1))^{2}<p n+o(1)$ (because $\left.p n<O(1)\right)$.

Our first technical lemma is a main lemma of this paper. This is a stronger version of the statement (3) of Lemma 1.

Lemma 2. W.h.p. we have

$$
\left|\|A \mathbf{1}-p n \mathbf{1}\|^{2}-\left(p-p^{2}\right) n^{2}\right| \leq p n^{1.5+\delta} .
$$

Hence, by choosing $\delta<0.5$, we immediately have

$$
|\|A \overline{\mathbf{1}}-p n \overline{\mathbf{1}}\|-\sqrt{p n}|<o(1) .
$$

As shown below, for proving these bounds, we do not need to modify a given random matrix by removing rows and columns with too many 1's as explained above. In other words, the modification was essential for proving (2) of Lemma 1. On the other hand, our real target matrix is $A^{\prime}$ that is obtained by removing rows of $V^{\prime}$ (and corresponding columns). Then we need bounds similar to the above for such $A^{\prime}$, and for this, the following corollary is given. Below let $\mathbf{1}^{\prime}=(1, \ldots, 1) / \sqrt{n^{\prime}}$. (Its proof, which idea is essentially given in $[3$, Proof of Lemma 39], is stated in Appendix.)

Corollary 3. W.h.p. we have

$$
\left|\| A^{\prime} \overline{\mathbf{1}}-p n^{\prime} \overline{\mathbf{1}^{\prime}}\right|\left|-\sqrt{p n^{\prime}}\right|<O\left(\left(p n^{\prime}\right)^{-2}\right)+o(1) .
$$

Now consider the proof of Lemma 2. Analysis of this type becomes much easier if we may assume that a given matrix is "completely random", i.e., all nondiagonal entries are iid random variables. On the other hand, our target random matrix $A$ is symmetric, and there are correlations between $a_{i j}$ and $a_{j i}$ for all pairs of $i$ and $j, 1 \leq i<j \leq n$. In fact, in the previous work [3, 4] some interesting techniques have been introduced to avoid such correlations. Here we take more direct approach and prove the lemma by considering these correlations. But in order to show the outline, we prove the lemma here ignoring the correlations; a tedious but rather straightforward argument for taking care the correlations is stated in Appendix.

Proof of Lemma 2 (Outline). For each $i \in[n]$, let $X_{i}=\sum_{j} a_{i j}$. Note that these are not iid random variables. But let us assume here that they were independent. (There is only one point in the whole proof where this independence 
is used, which will be reminded explicitly below.) Next we let $Y_{i}=\left(X_{i}-\mu\right)^{2}$, where $\mu=\mathrm{E}\left[X_{i}\right]=p(n-1)$, and let $Y=\sum_{i} Y_{i}$. Then we have

$$
\begin{aligned}
\mathrm{E}\left[Y_{i}\right] & =\mathrm{E}\left[X_{i}^{2}\right]-\mu^{2} \\
& =p(n-1)+p^{2}(n-1)(n-2)-p^{2}(n-1)^{2} \\
& =p(n-1)-p^{2}(n-1)=\left(p-p^{2}\right)(n-1) .
\end{aligned}
$$

Hence, we have $\mathrm{E}[Y]=\left(p-p^{2}\right) n(n-1)$.

On the other hand, following the argument of [3, Proof of Lemma 39], we have $\mathrm{V}\left[Y_{i}\right] \leq \mathrm{E}\left[Y_{i}^{2}\right]<O\left((p n)^{2}\right)$, which is bounded by $O(1)$ because $p n$ is a constant for our choice of $p$. Now if all $Y_{1}, \ldots, Y_{n}$ were independent, then we would have

$$
\mathrm{V}[Y]=\sum_{i} \mathrm{~V}\left[Y_{i}\right] \leq n \mathrm{E}\left[Y_{i}^{2}\right]<O(n) .
$$

Then by using Chebyshev bound, we can show that $Y$ is close to $\mathrm{E}[Y]$. More precisely, we have

$$
\begin{aligned}
\operatorname{Pr}\left[|Y-\mathrm{E}[Y]|>p n^{1.5+\delta}\right] & <\frac{\mathrm{V}[Y]}{\left(p n^{1.5+\delta}\right)^{2}} \\
& <\frac{O(n)}{\left(\Omega(1) n^{0.5+\delta}\right)^{2}}<o(1) .
\end{aligned}
$$

Note, on the other hand, that what we want to estimate is $\sum_{i}\left(X_{i}-p n\right)^{2}=$ $\sum_{i}\left(X_{i}-\mu-p\right)^{2}$. Let $Y_{i}^{\prime}=\left(X_{i}-\mu-p\right)^{2}$. Then noting

$$
\begin{aligned}
Y_{i}^{\prime} & =\left(X_{i}-\mu-p\right)^{2}=\left(X_{i}-\mu\right)^{2}-2 p\left(X_{i}-\mu\right)+p^{2} \\
& =Y_{i}-2 p\left(X_{i}-\mu\right)+p^{2}
\end{aligned}
$$

we have

$$
\mathrm{E}\left[Y_{i}^{\prime}\right]=\mathrm{E}\left[Y_{i}\right]+p^{2}=\left(p-p^{2}\right) n+\left(2 p^{2}-p\right) .
$$

Also we have

$$
\begin{aligned}
\mathrm{V}\left[Y_{i}^{\prime}\right] & =\mathrm{E}\left[\left(Y_{i}^{\prime}-\mathrm{E}\left[Y_{i}^{\prime}\right]\right)^{2}\right] \\
& =\mathrm{E}\left[\left(\left(Y_{i}-2 p\left(X_{i}-\mu\right)+p^{2}\right)-\left(\mathrm{E}\left[Y_{i}\right]+p^{2}\right)\right)^{2}\right] \\
& =\mathrm{E}\left[\left(\left(Y_{i}-\mathrm{E}\left[Y_{i}\right]\right)-2 p\left(X_{i}-\mu\right)\right)^{2}\right] \\
& =\mathrm{V}\left[Y_{i}\right]+4 p^{2} \mathrm{~V}\left[X_{i}\right] \\
& <O(1)+O\left(p^{2}\right)<O(1) .
\end{aligned}
$$

Hence, for $Y^{\prime}=\sum_{i} Y_{i}^{\prime}$, we can again show that $Y^{\prime}$ is close to $\mathrm{E}\left[Y^{\prime}\right]$ w.h.p., and from this we have

$$
\begin{aligned}
o(1) & >\operatorname{Pr}\left[\left|Y^{\prime}-\mathrm{E}\left(Y^{\prime}\right)\right|>2 p n^{1.5+\delta}\right] \\
& =\operatorname{Pr}\left[\left|Y^{\prime}-\left(\left(p-p^{2}\right) n^{2}+\left(2 p^{2}-p\right) n\right)\right|>2 p n^{1.5+\delta}\right] \\
& \geq \operatorname{Pr}\left[\left|Y^{\prime}-\left(p-p^{2}\right) n^{2}\right|>p n^{1.5+\delta}\right],
\end{aligned}
$$

where the last bound holds for any sufficiently large $n$. 
Finally consider the real situation where $Y_{1}, \ldots, Y_{n}$ are not independent. Even in this case, as shown in Appendix, we still have $\mathrm{V}[Y]<O(n)$ and similarly $\mathrm{V}\left[Y^{\prime}\right]<O(n)$. Therefore, essentially the same analysis as above is sufficient for the lemma.

Let us state some more small technical lemmas. In the following, let $A^{\prime}$ and $n^{\prime}$ be the random matrix and its size given in Lemma 1 , and let $\mathbf{1}^{\prime}$ denote the vector $(1, \ldots, 1)$ with $n^{\prime}$ components. We assume that the part (1) of Lemma 1 holds.

Lemma 4. For any unit vector $\boldsymbol{\xi}$ that is perpendicular to $\mathbf{1}^{\prime}$, and for any unit vector $\boldsymbol{\eta}$, w.h.p. we have

$$
\left|\left\langle A^{\prime} \boldsymbol{\xi}, \boldsymbol{\eta}\right\rangle\right| \leq c_{2} \sqrt{p n^{\prime}} .
$$

Proof. Let $A^{\prime} \boldsymbol{\xi}=\sum_{i} a_{i} \chi_{i}^{\prime}$, by using eigenvectors $\chi_{1}^{\prime}, \chi_{2}^{\prime}, \ldots, \chi_{n^{\prime}}^{\prime}$ of $A^{\prime}$, Then from Lemma 1 (2), w.h.p. we have

$$
\sum_{i} a_{i}^{2}=\left\|A^{\prime} \boldsymbol{\xi}\right\|^{2} \leq c_{2} p n^{\prime}
$$

On the other hand, letting $\boldsymbol{\eta}=\sum_{i} b_{i} \boldsymbol{\chi}^{\prime}{ }_{i}$, we have $\sum_{i} b_{i}^{2}=1$. Then the lemma is shown by

$$
\begin{aligned}
\left\langle A^{\prime} \boldsymbol{\xi}, \boldsymbol{\eta}\right\rangle^{2} & =\left\langle\sum_{i} a_{i} \boldsymbol{\chi}_{i}^{\prime}, \sum_{j} b_{j} \boldsymbol{\chi}_{j}^{\prime}\right\rangle^{2}=\left(\sum_{i} a_{i} b_{i}\right)^{2} \\
& \leq\left(\sum_{i} a_{i}^{2}\right)\left(\sum_{j} b_{j}^{2}\right) \leq\left(c_{2}\right)^{2} p n^{\prime} .
\end{aligned}
$$

By using this lemma, we can bound eigenvalues as follows.

Corollary 5. For some constant $c_{3}$ (e.g., letting $\left.c_{3}=3\right)$, w.h.p. we have

$$
\left|\lambda_{i}^{\prime}\right| \leq c_{3} c_{2} \sqrt{p n^{\prime}}
$$

for any $i, 2 \leq i \leq n^{\prime}$.

Remark. The coefficient $c_{3}=3$ is a bit loose. In fact, we have $\left|\lambda_{i}^{\prime}\right| \leq c_{2} \sqrt{p n^{\prime}}$ for all $i, 2 \leq i \leq n^{\prime}-1$.

Proof. We make use of the following Courant-Fischer characterization (recall that $\boldsymbol{\xi}$ denote a unit vector):

$$
\lambda_{i}^{\prime}=\max _{S: \operatorname{dim}(S)=i} \min _{\boldsymbol{\xi} \in S}\left\langle A^{\prime} \boldsymbol{\xi}, \boldsymbol{\xi}\right\rangle=\min _{S: \operatorname{dim}(S)=n^{\prime}-i+1} \max _{\boldsymbol{\xi} \in S}\left\langle A^{\prime} \boldsymbol{\xi}, \boldsymbol{\xi}\right\rangle .
$$

First for bounding $\lambda_{2}^{\prime}$ and $\lambda_{n-1}^{\prime}$, we use $S_{0}=\left\{\boldsymbol{v} \mid \boldsymbol{v} \perp \mathbf{1}^{\prime}\right\}$. Then w.h.p. we have

$$
\begin{aligned}
\lambda_{2}^{\prime} & \leq \max _{\boldsymbol{\xi} \in S_{0}}\langle A \boldsymbol{\xi}, \boldsymbol{\xi}\rangle \leq c_{2} \sqrt{p n^{\prime}}, \text { and } \\
\lambda_{n^{\prime}-1}^{\prime} & \geq \min _{\boldsymbol{\xi}_{\boldsymbol{\xi} \in S_{0}}}\langle A \boldsymbol{\xi}, \boldsymbol{\xi}\rangle \geq-c_{2} \sqrt{p n^{\prime}} .
\end{aligned}
$$


Finally for bounding $\lambda_{n^{\prime}}^{\prime}$, we let $\boldsymbol{\xi}_{*}$ to denote the one that defines $\lambda_{n}^{\prime}=$ $\left\langle A \boldsymbol{\xi}_{*}, \boldsymbol{\xi}_{*}\right\rangle$. Let $\boldsymbol{\xi}_{*}=a \overline{\mathbf{1}^{\prime}}+b \boldsymbol{\eta}$, where $a=\left\langle\boldsymbol{\xi}_{*}, \overline{\mathbf{1}^{\prime}}\right\rangle$ (and thus $|a|,|b| \leq 1$ and $\boldsymbol{\eta} \perp \overline{\mathbf{1}^{\prime}}$ ). Then by using (i) $\left\langle A^{\prime} \overline{\mathbf{1}^{\prime}}, \overline{\mathbf{1}^{\prime}}\right\rangle \geq 1$, (ii) the symmetry of $A^{\prime}$, and (iii) Lemma 4 , we have

$$
\begin{aligned}
\lambda_{n^{\prime}}^{\prime} & =\left\langle A^{\prime} \boldsymbol{\xi}_{*}, \boldsymbol{\xi}_{*}\right\rangle=\left\langle A^{\prime}\left(a \overline{\mathbf{1}^{\prime}}+b \boldsymbol{\eta}\right), a \overline{\mathbf{1}^{\prime}}+b \boldsymbol{\eta}\right\rangle \\
& =a^{2}\left\langle A^{\prime} \overline{\mathbf{1}^{\prime}}, \overline{\mathbf{1}^{\prime}}\right\rangle+a b\left(\left\langle A^{\prime} \boldsymbol{\eta}, \overline{\mathbf{1}^{\prime}}\right\rangle+\left\langle A^{\prime} \overline{\mathbf{1}^{\prime}}, \boldsymbol{\eta}\right\rangle\right)+b^{2}\left\langle A^{\prime} \boldsymbol{\eta}, \boldsymbol{\eta}\right\rangle \\
& =a^{2}\left\langle A^{\prime} \overline{\mathbf{1}^{\prime}}, \overline{\mathbf{1}^{\prime}}\right\rangle+2 a b\left(\left\langle A^{\prime} \boldsymbol{\eta}, \overline{\mathbf{1}^{\prime}}\right\rangle+b^{2}\left\langle A^{\prime} \boldsymbol{\eta}, \boldsymbol{\eta}\right\rangle\right. \\
& \geq-\left(2 a b+b^{2}\right) \sqrt{p n^{\prime}},
\end{aligned}
$$

which is our desired bound.

On the other hand, since $\left\langle A^{\prime} \mathbf{1}^{\prime}, \mathbf{1}^{\prime}\right\rangle$ is $\sum_{i j} a_{i j}$, we have the following from the Chernoff bound. This shows that $\lambda_{1}^{\prime}>p n^{\prime}-o(1)$.

Lemma 6. For any $\delta, 0<\delta \leq 1 / 2$, w.h.p. we have

$$
p n^{\prime}-p n^{\prime 1-\delta} \leq\left\langle A^{\prime} \overline{\mathbf{1}^{\prime}}, \overline{\mathbf{1}^{\prime}}\right\rangle \leq p n^{\prime}+p n^{\prime 1-\delta} .
$$

\section{MAin ANALYSiS}

As explained above, we will analyze matrix $A^{\prime}$ that is defined in Lemma 1 by removing rows of $V^{\prime \prime}$ (and the corresponding columns) from matrix $A$ generated randomly with the density parameter $p$. We may assume that Lemma 1 (1) holds. For simplifying our notation, throughout this section we will use $A$ and $n$ (instead of $A^{\prime}$ and $n^{\prime}$ ) to denote a random matrix of this type and its size respectively. Also throughout this section, we assume that $\alpha$ is sufficiently large, or, more specifically, $\alpha>\left(c_{2}\right)^{2}$, where $c_{2}$ is the constant of Lemma 1 .

For stating our result succinctly we introduce some more order notations. By $o_{\alpha}(1)$ and $o_{\alpha^{+}}(1)$ we denote some positive functions $\varepsilon_{1}(\alpha)$ and $\varepsilon_{2}(\alpha)$ that are defined in each context to satisfy $\varepsilon_{1}(\alpha) \leq d_{1} / \alpha^{b_{1}}$ and $\varepsilon_{2}(\alpha) \leq d_{2} / \alpha^{1.5}$ respectively with some constants $b_{1}, d_{1}, d_{2}>0$ independent from $n$ and $\alpha$. That is, while $o_{\alpha}(1)$ goes to 0 when $\alpha$ goes $\infty, o_{\alpha^{+}}(1)$ is a function that goes to 0 faster than $1 / \alpha$. For any positive function $f(\alpha)$, if it satisfies that $\exists c>0, \alpha_{0}>0, \forall \alpha>\alpha_{0}$, then we have

$$
\begin{aligned}
& \frac{1}{f(\alpha)-o_{\alpha}(1)} \leq \frac{1}{f(\alpha)}+o_{\alpha}(1), \text { and } \\
& \frac{1}{f(\alpha)+o_{\alpha}(1)} \geq \frac{1}{f(\alpha)}-o_{\alpha}(1) .
\end{aligned}
$$

These derivations are useful in the following analysis. With this notation, the bound of Corollary 3 can be restated as

$$
|\|A \overline{\mathbf{1}}-p n \overline{\mathbf{1}}\|-\sqrt{p n}|<o_{\alpha^{+}}(1)+o(1)
$$


What is essential for our analysis is the relation between $\chi_{1}$ and $\overline{\mathbf{1}}$. In the following we fix $a_{1}, \ldots, a_{n}, b$, and $\chi$ to those satisfying

$$
\overline{\mathbf{1}}=a_{1} \boldsymbol{\chi}_{1}+\cdots+a_{n} \boldsymbol{\chi}_{n}=a_{1} \boldsymbol{\chi}_{1}+b \boldsymbol{\chi} .
$$

Here recall that the overlap $a$ is defined by $a=\left\langle\chi_{1}, \overline{\mathbf{1}}\right\rangle$. Hence, we have $a_{1}=a$ and

$$
\chi_{1}=a \overline{\mathbf{1}}+b \boldsymbol{\eta}
$$

for some $\boldsymbol{\eta}$ such that $\boldsymbol{\eta} \perp \overline{\mathbf{1}}$. Note that $a^{2}+b^{2}=1$ and $b^{2}=\sum_{i>2} a_{i}^{2}$. Thus, for bounding $a$ (which is one of our goals), we will estimate $\sum_{i \geq 2} a_{i}^{2}\left(=b^{2}\right)$ and $\sqrt{1-b^{2}}(=a)$.

Here the key tool is the bound (2) applied to the following relation:

$$
\begin{aligned}
\|A \overline{\mathbf{1}}-p n \overline{\mathbf{1}}\|^{2} & =\left\|A\left(\sum_{i} a_{i} \boldsymbol{\chi}_{i}\right)-p n\left(\sum_{i} a_{i} \boldsymbol{\chi}_{i}\right)\right\|^{2} \\
& =\left\|\sum_{i}\left(a_{i} \lambda_{i}-a_{i} \cdot p n\right) \boldsymbol{\chi}_{i}\right\|^{2} \\
& =\sum_{i} a_{i}^{2}\left(\lambda_{i}-p n\right)^{2} .
\end{aligned}
$$

Theorem 1. W.h.p. we have

$$
a \geq \sqrt{1-\frac{1}{\alpha}-o_{\alpha^{+}}(1)-o(1)}
$$

Proof. By using (2) and (3), we have

$$
\begin{aligned}
p n & +o_{\alpha^{+}}(1)+o(1) \\
& >\sum_{i} a_{i}^{2}\left(\lambda_{i}-p n\right)^{2} \geq \sum_{i \geq 2} a_{i}^{2}\left(\lambda_{i}-p n\right)^{2} \\
\geq & \sum_{i \geq 2} a_{i}^{2}\left(c_{2} \sqrt{p n}-p n\right)^{2}=\sum_{i \geq 2} a_{i}^{2}\left(\sqrt{p n}-c_{2}\right)^{2} p n .
\end{aligned}
$$

Hence,

$$
b^{2}=\sum_{i \geq 2} a_{i}^{2}<\frac{1+o_{\alpha^{+}}(1)+o(1)}{\left(\sqrt{p n}-c_{2}\right)^{2}}<\frac{1}{\left(\sqrt{\alpha}-c_{2}\right)^{2}}+o_{\alpha^{+}}(1)+o(1),
$$

and then our desired bound (4) follows.

Next we give the following upper bound for $\lambda_{1}$.

Theorem 2. W.h.p. we have

$$
\lambda_{1}<\alpha+1+o_{\alpha}(1)+o(1) .
$$


Proof. We first show that

$$
\lambda_{1}<\alpha+\frac{\sqrt{\alpha}}{a\left(\sqrt{\alpha}-c_{2}\right)}+o_{\alpha^{+}}(1)+o(1)
$$

holds w.h.p. Let $\boldsymbol{w}=A \overline{\mathbf{1}}-p n \overline{\mathbf{1}}$. We estimate $\left\langle A \overline{\mathbf{1}}, \boldsymbol{\chi}_{1}\right\rangle$ in the following two ways:

$$
\begin{aligned}
\left\langle A \overline{\mathbf{1}}, \boldsymbol{\chi}_{1}\right\rangle & =\left\langle A\left(a \boldsymbol{\chi}_{1}+b \boldsymbol{\chi}\right), \boldsymbol{\chi}_{1}\right\rangle \\
& =\left\langle a \lambda_{1} \boldsymbol{\chi}_{1}+b A \boldsymbol{\chi}, \boldsymbol{\chi}_{1}\right\rangle=a \lambda_{1}, \text { and } \\
\left\langle A \overline{\mathbf{1}}, \boldsymbol{\chi}_{1}\right\rangle & =\langle A \overline{\mathbf{1}}, a \overline{\mathbf{1}}+b \boldsymbol{\eta}\rangle \\
& =a\langle A \overline{\mathbf{1}}, \overline{\mathbf{1}}\rangle+b\langle p n \overline{\mathbf{1}}+\boldsymbol{w}, \boldsymbol{\eta}\rangle \\
& =a\langle A \overline{\mathbf{1}}, \overline{\mathbf{1}}\rangle+b\langle\boldsymbol{w}, \boldsymbol{\eta}\rangle \leq a\langle A \overline{\mathbf{1}}, \overline{\mathbf{1}}\rangle+b\|\boldsymbol{w}\| \\
& <a\langle A \overline{\mathbf{1}}, \overline{\mathbf{1}}\rangle+b \sqrt{p n}+o_{\alpha^{+}}(1)+o(1) \quad(\text { by }(2) \text { and }|b| \leq 1) \\
& <a\langle A \overline{\mathbf{1}}, \overline{\mathbf{1}}\rangle+\frac{\sqrt{p n}}{\sqrt{p n}-c_{2}}+o_{\alpha^{+}}(1)+o(1) . \quad(\text { by }(5))
\end{aligned}
$$

The bound (7) follows from these two bounds.

Next we derive (6). First note that from (4) we have

$$
\frac{1}{a^{2}}<\left(1+\frac{1}{\alpha}+o_{\alpha^{+}}(1)+o(1)\right)^{2} .
$$

Using this bound, the desired bound is shown by

$$
\begin{aligned}
\lambda_{1} & <\alpha+\frac{1}{a} \cdot \frac{\sqrt{\alpha}}{\sqrt{\alpha}-c_{2}}+o_{\alpha^{+}}(1)+o(1) \\
& <\alpha+\frac{1}{a}\left(1+o_{\alpha}(1)\right)+o_{\alpha^{+}}(1)+o(1) \\
& <\alpha+\left(1+\frac{1}{\alpha}+o_{\alpha^{+}}(1)+o(1)\right)\left(1+o_{\alpha}(1)\right) \\
& <\alpha+1+o_{\alpha}(1)+o(1) .
\end{aligned}
$$

Theorem 3. W.h.p. we have

$$
a<\sqrt{1-\frac{1}{\alpha}+o_{\alpha^{+}}(1)+o(1)}
$$

Thus, within our approximation, this upper bound matches to the lower bound (4). 
Proof. Here again using (2) and (3), we have

$$
\begin{aligned}
p n-o_{\alpha^{+}}(1)-o(1) & <\sum_{i} a_{i}^{2}\left(\lambda_{i}-p n\right)^{2} \\
& =a^{2}\left(\lambda_{1}-p n\right)^{2}+\sum_{i \geq 2} a_{i}^{2}\left(\lambda_{i}-p n\right)^{2} \\
& <\frac{p n}{\left(\sqrt{p n}-c_{2}\right)^{2}}+o_{\alpha^{+}}(1)+o(1)+\sum_{i \geq 2} a_{i}^{2}\left(p n+c_{3} c_{2} \sqrt{p n}\right)^{2} .
\end{aligned}
$$

Here for deriving the last inequality, we used bounds for $\lambda_{1}$ : a lower bound $p n-o(1)$ is from Lemma 6, and an upper bound is (7).

Hence we have

$$
\begin{aligned}
b^{2} & =\sum_{i \geq 2} a_{i}^{2}>\frac{1-\left(\sqrt{p n}-c_{2}\right)^{-2}}{\left(c_{2} c_{3}+\sqrt{p n}\right)^{2}}-o_{\alpha^{+}}(1)-o(1) \\
& =\frac{1-\left(\sqrt{\alpha}-c_{2}\right)^{-2}}{\left(c_{2} c_{3}+\sqrt{\alpha}\right)^{2}}-o_{\alpha^{+}}(1)-o(1) .
\end{aligned}
$$

Hence,

$$
a<\sqrt{1-\frac{1-\left(\sqrt{\alpha}-c_{2}\right)^{-2}}{\left(c_{2} c_{3}+\sqrt{\alpha}\right)^{2}}+o_{\alpha^{+}}(1)+o(1)} .
$$

Then the bound (8) of the lemma follows.

Finally we show the following lower bound for $\lambda_{1}$.

Theorem 4. W.h.p. we have

$$
\lambda_{1}>\alpha+1-o_{\alpha}(1)-o(1) .
$$

Proof. We start by estimating $\|A \overline{\mathbf{1}}\|^{2}$. Let $\boldsymbol{w}^{\prime}=A \overline{\mathbf{1}}-\langle A \overline{\mathbf{1}}, \overline{\mathbf{1}}\rangle \overline{\mathbf{1}}$. Then since $\boldsymbol{w}^{\prime} \perp \overline{\mathbf{1}}$, we have

$$
\begin{aligned}
\|A \overline{\mathbf{1}}\|^{2} & =\langle A \overline{\mathbf{1}}, \overline{\mathbf{1}}\rangle^{2}+\left\|\boldsymbol{w}^{\prime}\right\|^{2} \geq\left(p n-p n^{1-\delta}\right)^{2}+\left\|\boldsymbol{w}^{\prime}\right\|^{2} \\
& >(p n)^{2}+\left\|\boldsymbol{w}^{\prime}\right\|^{2}-o(1) .
\end{aligned}
$$

On the other hand, we let $\boldsymbol{w}=A \overline{\mathbf{1}}-p n \overline{\mathbf{1}}$, and then we have

$$
\boldsymbol{w}^{\prime}+(\langle A \overline{\mathbf{1}}, \overline{\mathbf{1}}\rangle-p n) \overline{\mathbf{1}}=\boldsymbol{w} .
$$

Hence, by the triangular inequality, we have

$$
\begin{aligned}
\left\|\boldsymbol{w}^{\prime}\right\| & \geq\|\boldsymbol{w}\|-\|(\langle A \overline{\mathbf{1}}, \overline{\mathbf{1}}\rangle \overline{\mathbf{1}}-p n) \overline{\mathbf{1}}\| \\
& =\|A \overline{\mathbf{1}}-p n \overline{\mathbf{1}}\|-\|(\langle A \overline{\mathbf{1}}, \overline{\mathbf{1}}\rangle \overline{\mathbf{1}}-p n) \overline{\mathbf{1}}\| \\
& >\left(\sqrt{p n}-o_{\alpha^{+}}(1)-o(1)\right)-\left\|\left(p n^{1-\delta}\right) \overline{\mathbf{1}}\right\|,
\end{aligned}
$$


where (2) and Lemma 6 are used to get this bound for some $\delta>0$. Thus, we have

$$
\left\|\boldsymbol{w}^{\prime}\right\|>\sqrt{p n}-o_{\alpha^{+}}(1)-o(1) .
$$

Then by (10), we have

$$
\|A \overline{\mathbf{1}}\|^{2}>(p n)^{2}+p n-o_{\alpha^{+}}(1)-o(1) .
$$

On the other hand, we have

$$
\|A \overline{\mathbf{1}}\|^{2}=a^{2} \lambda_{1}^{2}+\sum_{i \geq 2} a_{i}^{2} \lambda_{i}^{2} \leq a^{2} \lambda_{1}^{2}+b^{2}\left(c_{3} c_{2}\right)^{2} p n .
$$

Hence, by using (11), we have

$$
\begin{aligned}
\lambda_{1}^{2} & >\frac{1}{a^{2}} \cdot(p n)^{2}+p n-b^{2}\left(c_{3} c_{2}\right)^{2} p n-o_{\alpha^{+}}(1)-o(1) \\
& =\frac{\alpha^{2}}{a^{2}}+\alpha-b^{2}\left(c_{3} c_{2}\right)^{2} \alpha-o_{\alpha^{+}}(1)-o(1) .
\end{aligned}
$$

Now we analyze below the first and the third terms of this bound. For the third term, by using the upper bound (5), we can show some constant $d_{1}>0$ exists such that

$$
\begin{aligned}
b^{2}\left(c_{3} c_{2}\right)^{2} \alpha & >\left(\frac{1}{\alpha}+o_{\alpha^{+}}(1)+o(1)\right)\left(c_{2} c_{2}\right)^{2} \alpha \\
& >d_{1}+o_{\alpha}(1)+o(1)
\end{aligned}
$$

holds. On the other hand, by using (8), we have some $d_{2}$ with which the first term is bounded by

$$
\begin{aligned}
\frac{\alpha^{2}}{a^{2}} & \geq \frac{1}{1-1 / \alpha+o_{\alpha^{+}}(1)+o(1)} \cdot \alpha^{2} \\
& =\left(1+\frac{1-\alpha \cdot o_{\alpha^{+}}(1)}{\alpha-1+\alpha \cdot o_{\alpha^{+}}(1)}\right) \cdot \alpha^{2} \\
& \geq \alpha^{2}+\frac{\alpha^{2}-\alpha^{3} \cdot o_{\alpha^{+}}(1)}{\alpha+\alpha \cdot o_{\alpha^{+}}(1)} \\
& >\alpha^{2}+\alpha\left(\frac{1}{1+o_{\alpha^{+}}(1)}\right)-d_{2} \alpha^{-0.5}
\end{aligned}
$$

From these bounds, for any $\epsilon>0$, we have

$$
\begin{aligned}
\lambda_{1}^{2} \geq \alpha^{2} & +2 \alpha\left(\frac{1-\epsilon}{1+o_{\alpha^{+}}(1)}\right)+\left(\frac{1-\epsilon}{1+o_{\alpha^{+}}(1)}\right)^{2} \\
& +\frac{2 \alpha \epsilon}{1+o_{\alpha^{+}}(1)}-\left(\frac{1-\epsilon}{1+o_{\alpha^{+}}(1)}\right)^{2}-d_{1}-d_{2} \alpha^{-0.5}-o_{\alpha^{+}}(1)-o(1),
\end{aligned}
$$

where the sum of the last six terms is positive by taking $\epsilon=d_{3} \alpha^{-1.5}+o_{\alpha^{+}}(1)+o(1)$ for some sufficiently large constant $d_{3}>0$. 
Therefore, with this $d_{3}$, we have

$$
\lambda_{1}>\alpha+\left(\frac{1-d_{3} \alpha^{-1.5}-o_{\alpha^{+}}(1)-o(1)}{1+o_{\alpha^{+}}(1)}\right)>\alpha+1-o_{\alpha^{+}}(1)-o(1) .
$$

\section{REFERENCES}

[1] N. Alon, Spectral techniques in graph algorithms, in Proc. the 3rd Latin American Symposium (LATIN'98), Lecture Notes in Computer Science 1380, Springer, 206-215, 1998.

[2] N. Alon and N. Kahale, A spectral technique for coloring random 3-colorable graphs, SIAM J. Computing 26(6) (1997), 1733-1748.

[3] A. Coja-Oghlan, A. Goerdt, A. Lanka, and F. Schädlich, Techniques from combinatorial approximation algorithms yield efficient algorithms for random $2 k$-SAT, Theoretical Computer Science 329 (2004), 1-45.

[4] U. Feige and E. Ofek, Spectral techniques applied to sparse random graphs, Random Structures and Algorithms 27 (2005), 251-275.

[5] J. Friedman, J. Kahn, and E. Szemeredi, On the second eigenvalue in random regular graphs, in Proc 21st Annu. ACM Sympos. Theory of Computing (STOC'89), 587-598, 1989.

[6] Z. Furedi and J. Komlos, The eigenvalues of random symmetric matrices, Combinatorica 1(3) (1981), 233-241.

[7] Y. Kabashima, H. Takahashi, and O. Watanabe, Cavity approach to the first eigenvalue problem in a family of symmetric random sparse matrices, arXiv:1001.3935, to appear in Proceedings of IW-SMI2010.

[8] M. Krivelevich and B. Sudakov, The largest eigenvalue of sparse random graphs, Combinatorics, Probability and Computing 12 (2003), 61-72.

\section{ApPEndix}

A. Analysis of $\mathrm{V}[Y]$. Here we give a detail analysis of $\mathrm{V}[Y]$ that is omitted in the proof of Lemma 2. We use the same notations, but let us recall important ones.

$$
\begin{aligned}
X_{i} & =\sum_{j} a_{i j}, \quad \mu=\mathrm{E}\left[X_{i}\right]=p(n-1)<O(1), \\
Y_{i} & =\left(X_{i}-\mu\right)^{2}, \text { and } Y=\sum_{i} Y_{i} .
\end{aligned}
$$

For simplifying our notation, we introduce here the following notations:

$$
\begin{aligned}
& E_{\mathrm{X} 2}=\mathrm{E}\left[X_{1}^{2}\right]=p(n-1)+p^{2}(n-1)(n-2), \\
& E_{\mathrm{Y} 1}=\mathrm{E}\left[Y_{1}\right]=\mathrm{E}\left[\left(X_{1}-\mu\right)^{2}\right]=E_{\mathrm{X} 2}-\mu^{2}, \text { and } \\
& E_{\mathrm{Y} 2}=\mathrm{E}\left[Y_{1}^{2}\right]<O\left((p n)^{2}\right)<O(1) .
\end{aligned}
$$

By symmetry we have $E_{\mathrm{X} 2}=\mathrm{E}\left[X_{i}^{2}\right], E_{\mathrm{Y} 1}=\mathrm{E}\left[Y_{i}\right]$, and $E_{\mathrm{Y} 2}=\mathrm{E}\left[Y_{i}^{2}\right]$ for all $i \in[n]$. 
Our goal is to estimate $\mathrm{V}[Y]$. Let $\nu=\mathrm{E}[Y]$; note that $\nu=n E_{\mathrm{Y} 1}$. First by definition we have

$$
\begin{aligned}
& \mathrm{V}[Y]=\mathrm{E}\left[(Y-\nu)^{2}\right]=\mathrm{E}\left[Y^{2}-2 Y \nu+\nu^{2}\right] \\
& =\mathrm{E}\left[\left(\sum_{i} Y_{i}\right)^{2}\right]-2 \mathrm{E}[Y] \nu+\nu^{2}=\mathrm{E}\left[\left(\sum_{i} Y_{i}\right)^{2}\right]-\nu^{2} \\
& =\mathrm{E}\left[\sum_{i} Y_{i}^{2}\right]+\mathrm{E}\left[\sum_{i \neq i^{\prime}} Y_{i} \cdot Y_{i^{\prime}}\right]-\nu^{2} \\
& =n E_{\mathrm{Y} 2}+n(n-1) \mathrm{E}\left[Y_{1} \cdot Y_{2}\right]-n^{2}\left(E_{\mathrm{Y} 1}\right)^{2} \\
& \leq n E_{\mathrm{Y} 2}+n^{2}\left(\mathrm{E}\left[Y_{1} \cdot Y_{2}\right]-\left(E_{\mathrm{Y} 1}\right)^{2}\right),
\end{aligned}
$$

where by symmetry the choice of $Y_{1}$ and $Y_{2}$ is not essential.

If we could assume that $Y_{1}$ and $Y_{2}$ are independent, then we would have

$$
(13)=n E_{\mathrm{Y} 2}+n^{2}\left(\mathrm{E}\left[Y_{1}\right] \cdot \mathrm{E}\left[Y_{2}\right]-\left(E_{\mathrm{Y} 1}\right)^{2}\right)=n E_{\mathrm{Y} 2}
$$

Thus, for our estimation, we consider the difference between $\mathrm{E}\left[Y_{1} \cdot Y_{2}\right]$ and $\left(E_{\mathrm{Y} 1}\right)^{2}$.

Again by definition, we have

$$
\begin{aligned}
& \mathrm{E}\left[Y_{1} \cdot Y_{2}\right] \\
&= \mathrm{E}\left[\left(X_{1}-\mu\right)^{2}\left(X_{2}-\mu\right)^{2}\right] \\
&= \mathrm{E}\left[\left(X_{1}^{2}-2 \mu X_{1}+\mu^{2}\right)\left(X_{2}^{2}-2 \mu X_{2}+\mu^{2}\right)\right] \\
&= \mathrm{E}\left[X_{1}^{2} X_{2}^{2}-2 \mu X_{1}^{2} X_{2}+\mu^{2} X_{1}^{2}\right. \\
&\left.\quad \quad-2 \mu X_{1} X_{2}^{2}+4 \mu^{2} X_{1} X_{2}-2 \mu^{3} X_{1}+\mu^{2} X_{2}^{2}-2 \mu^{3} X_{2}+\mu^{4}\right] \\
&= \mathrm{E}\left[X_{1}^{2} X_{2}^{2}\right]-4 \mu \mathrm{E}\left[X_{1}^{2} X_{2}\right]+4 \mu^{2} \mathrm{E}\left[X_{1} X_{2}\right]+2 \mu^{2} E_{\mathrm{X} 2}-4 \mu^{4}+\mu^{4} .
\end{aligned}
$$

We estimate expectations $\mathrm{E}\left[X_{1}^{2} X_{2}^{2}\right], \mathrm{E}\left[X_{1}^{2} X_{1}\right]$, and $\mathrm{E}\left[X_{1} X_{2}\right]$.

First consider $\mathrm{E}\left[X_{1} X_{2}\right]$. Since $X_{1}=\sum_{j} a_{1 j}$ and $X_{2}=\sum_{j} a_{2 j}$, the correlation between $X_{1}$ and $X_{2}$ is caused only by $a_{12}=a_{21}$; all the other variables are independent of each other. In the following, let us use the following notations:

$$
S_{\mathrm{a} 1}=\sum_{j \neq 2} a_{1 j}, \text { and } S_{\mathrm{a} 2}=\sum_{j \neq 1} a_{2 j} .
$$

Also let $a^{\prime}$ denote $a_{12}=a_{21}$. Hence, we have $X_{1}=S_{\mathrm{a} 1}+a^{\prime}$ and $X_{2}=S_{\mathrm{a} 2}+a^{\prime}$, and it holds that

$$
\begin{aligned}
\mathrm{E}\left[X_{1} X_{2}\right] & =\mathrm{E}\left[\left(S_{\mathrm{a} 1}+a^{\prime}\right)\left(S_{\mathrm{a} 2}+a^{\prime}\right)\right] \\
& =\mathrm{E}\left[S_{\mathrm{a} 1} S_{\mathrm{a} 2}\right]+2 \mathrm{E}\left[a^{\prime} S_{\mathrm{a} 1}\right]+\mathrm{E}\left[a^{\prime 2}\right] \\
& =(p(n-2))^{2}+2 p(p(n-2))+p \\
& =p^{2} n(n-2)+p=\mu^{2}-p^{2}+p .
\end{aligned}
$$


Next consider $\mathrm{E}\left[X_{1}^{2} X_{2}\right]$. Again using our notations, we can evaluate it as

$$
\begin{aligned}
\mathrm{E}\left[X_{1}^{2} X_{2}\right] & =\mathrm{E}\left[X_{1}^{2} S_{\mathrm{a} 2}+\left(S_{\mathrm{a} 1}+a^{\prime}\right)^{2} a^{\prime}\right] \\
& =E_{\mathrm{X} 2} \cdot \mathrm{E}\left[S_{\mathrm{a} 2}\right]+\mathrm{E}\left[a^{\prime}\left(S_{\mathrm{a} 1}\right)^{2}\right]+\mathrm{E}\left[2 a^{\prime 2} S_{\mathrm{a} 1}\right]+\mathrm{E}\left[a^{\prime 3}\right] \\
& =E_{\mathrm{X} 2} \cdot(\mu-p)+p\left(p^{2}(n-2)(n-3)+3 p(n-2)+1\right)
\end{aligned}
$$

Here we compare the last term of (16) and

$$
E_{\mathrm{X} 2}=p(n-1)+p^{2}(n-1)(n-2) . \quad(\text { by }(12))
$$

Then we have

$$
\begin{aligned}
(16) & \left.=E_{\mathrm{X} 2} \cdot(\mu-p)+p\left(E_{\mathrm{X} 2}+2 p n+1-2 p^{2}(n-2)-5 p\right)\right) \\
& =E_{\mathrm{X} 2} \cdot \mu+2 p^{2} n+p-2 p^{3}(n-2)-5 p^{2} .
\end{aligned}
$$

The last one is $\mathrm{E}\left[X_{1}^{2} X_{2}^{2}\right]$. This can be stated as

$$
\begin{aligned}
\mathrm{E}\left[X_{1}^{2} X_{2}^{2}\right]= & \mathrm{E}\left[X_{1}^{2}\left(S_{\mathrm{a} 2}+a^{\prime}\right)^{2}\right] \\
= & \mathrm{E}\left[X_{1}^{2}\left(\left(S_{\mathrm{a} 2}\right)^{2}+2 a^{\prime} S_{\mathrm{a} 2}+a^{\prime 2}\right)\right] \\
= & E_{\mathrm{X} 2} \cdot \mathrm{E}\left[\left(S_{\mathrm{a} 2}\right)^{2}\right]+\mathrm{E}\left[\left(\left(S_{\mathrm{a} 1}\right)^{2}+2 a^{\prime} S_{\mathrm{a} 1}+a^{\prime 2}\right)\left(2 a^{\prime} S_{\mathrm{a} 2}+a^{\prime 2}\right)\right] \\
= & E_{\mathrm{X} 2} \cdot \mathrm{E}\left[\left(S_{\mathrm{a} 2}\right)^{2}\right] \\
& \quad+\mathrm{E}\left[2 a^{\prime}\left(S_{\mathrm{a} 1}\right)^{2} S_{\mathrm{a} 2}\right]+\mathrm{E}\left[a^{\prime 2}\left(S_{\mathrm{a} 1}\right)^{2}\right] \\
& \quad+\mathrm{E}\left[4 a^{\prime 2}\left(S_{\mathrm{a} 1}\right)\left(S_{\mathrm{a} 2}\right)\right]+\mathrm{E}\left[2 a^{\prime 3} S_{\mathrm{a} 1}\right] \\
& \quad+\mathrm{E}\left[2 a^{\prime 3} S_{\mathrm{a} 2}\right]+\mathrm{E}\left[a^{\prime 4}\right] \\
= & E_{\mathrm{X} 2} \cdot E_{X 2}^{\prime}+2 p \mu^{\prime} E_{X 2}^{\prime}+p E_{X 2}^{\prime}+4 p \mu^{\prime 2}+4 p \mu^{\prime}+p,
\end{aligned}
$$

where we let $E_{X 2}^{\prime}$ and $\mu^{\prime}$ denote $\mathrm{E}\left[\left(S_{\mathrm{a} 1}\right)^{2}\right]\left(=\mathrm{E}\left[\left(S_{\mathrm{a} 2}\right)^{2}\right]\right)$ and $\mathrm{E}\left[S_{\mathrm{a} 1}\right]\left(=\mathrm{E}\left[S_{\mathrm{a} 2}\right]\right)$ respectively.

Here note that

$$
E_{\mathrm{X} 2}=\mathrm{E}\left[X_{1}^{2}\right]=\mathrm{E}\left[\left(S_{\mathrm{a} 1}^{2}+2 a^{\prime} S_{\mathrm{a} 1}+a^{\prime 2}\right]=E_{X 2}^{\prime}+2 p \mu^{\prime}+p .\right.
$$

Hence $E_{\mathrm{X} 2} \cdot E_{\mathrm{X} 2}$ is expressed as

$$
\begin{aligned}
E_{\mathrm{X} 2} \cdot E_{\mathrm{X} 2} & =E_{\mathrm{X} 2} \cdot\left(E_{X 2}^{\prime}+2 p \mu^{\prime}+p\right) \\
& =E_{\mathrm{X} 2} \cdot E_{X 2}^{\prime}+\left(E_{X 2}^{\prime}+2 p \mu^{\prime}+p\right)\left(2 p \mu^{\prime}+p\right) \\
& =E_{\mathrm{X} 2} \cdot E_{X 2}^{\prime}+2 p \mu^{\prime} E_{X 2}^{\prime}+p E_{X 2}^{\prime}+4 p^{2} \mu^{\prime 2}+2 p^{2} \mu^{\prime}+p^{2} .
\end{aligned}
$$

Comparing this with (16), we can easily see that

$$
\mathrm{E}\left[X_{1}^{2} X_{2}^{2}\right]=\left(E_{\mathrm{X} 2}\right)^{2}+p\left(4 \mu^{\prime 2}+4 \mu^{\prime}+1\right)-p^{2}\left(4 \mu^{\prime 2}+2 \mu^{\prime}+1\right)
$$

Now by (15), (16), and (16), we can restate (14) as

$$
\begin{aligned}
& \mathrm{E}\left[Y_{1} \cdot Y_{2}\right] \quad(=(14)) \\
& \quad \leq\left(E_{\mathrm{X} 2}\right)^{2}-2 \mu^{2} E_{\mathrm{X} 2}+\mu^{4}+p\left(4 \mu^{\prime 2}+4 \mu^{\prime}+1\right)+4 \mu\left(2 p^{3}(n-2)+5 p^{2}\right)+4 \mu^{2} p .
\end{aligned}
$$


On the other hand, since we have

$$
\left(E_{\mathrm{Y} 1}\right)^{2}=\left(E_{\mathrm{X} 2}-\mu^{2}\right)^{2}=\left(E_{\mathrm{X} 2}\right)^{2}-2 \mu^{2} E_{\mathrm{X} 2}+\mu^{4},
$$

and $p n=\alpha<O(1)$, it holds that

$$
\mathrm{E}\left[Y_{1} \cdot Y_{2}\right]<\mathrm{E}\left[Y_{1}\right] \cdot \mathrm{E}\left[Y_{2}\right]+p \cdot O(1)
$$

which implies

$$
\mathrm{V}[Y]<n E_{\mathrm{Y} 2}+n^{2} p \cdot O(1)<O(n) .
$$

This is our desired bound.

B. A proof of Corollary 3. Recall that $A$ is an $n$-by- $n$ symmetric matrix while $A^{\prime}$ is $n^{\prime}$-by- $n^{\prime}$ (and symmetric). Also recall that we use $\mathbf{1}$ and $\mathbf{1}^{\prime}$ to denote all one vectors with $n$ components and $n^{\prime}$ components respectively, and that $\overline{\mathbf{1}}$ and $\overline{\mathbf{1}^{\prime}}$ are the corresponding normalized verctors.

We note two facts used here: ||$|A \overline{\mathbf{1}}-p n \overline{\mathbf{1}}||-\sqrt{p n}|=o(1)$, and $n^{\prime} \geq$ $\left(1-\exp \left(-p n / c_{2}\right)\right) n$. As a first step to showing Corollary 3 , we derive the following bound:

$$
\begin{aligned}
& \left\|\left|A^{\prime} \overline{\mathbf{1}^{\prime}}-p n \overline{\mathbf{1}^{\prime}} \|-\sqrt{p n}\right|\right. \\
& \quad \leq\left\|A^{\prime} \overline{\mathbf{1}^{\prime}}-p n \overline{\mathbf{1}^{\prime}}\right\|-\|A \overline{\mathbf{1}}-p n \overline{\mathbf{1}}\||+|\|A \overline{\mathbf{1}}-p n \overline{\mathbf{1}}\|-\sqrt{p n} \mid \\
& \quad \leq\left\|A^{\prime} \overline{\mathbf{1}^{\prime}}-p n \overline{\mathbf{1}^{\prime}}\right\|-\|A \overline{\mathbf{1}}-p n \overline{\mathbf{1}}\| \mid+o(1) .
\end{aligned}
$$

For the first term of the last formula above, we invoke a claim from [3], precisely the formula (28) therein. That is,

$$
\forall S \subset V:|S| \leq(p n)^{-10} n\left[\sum_{v \in V} \sum_{u \in S} a_{v u} \leq(p n)^{-5} n\right] .
$$

Let $x_{v}=\sum_{u \in V^{\prime}} a_{v u}$ and $y_{v}=\sum_{u \in V^{\prime \prime}} a_{v u}$. Then, $\sum_{v \in V^{\prime}} y_{v} \leq(p n)^{-5} n$. Thus,

$$
\begin{aligned}
\left\|A^{\prime} \mathbf{1}^{\prime}-p n \mathbf{1}^{\prime}\right\|^{2}-\|A \mathbf{1}-p n \mathbf{1}\|^{2} & =\sum_{v \in V^{\prime}}\left(x_{v}-p n\right)^{2}-\sum_{v \in V}\left(x_{v}+y_{v}-p n\right)^{2} \\
& \leq \sum_{v \in V^{\prime}}\left(\left(x_{v}-p n\right)^{2}-\left(x_{v}+y_{v}-p n\right)^{2}\right) \\
& \leq \sum_{v \in V^{\prime}} 2 p n y_{v} \leq 2 p n(p n)^{-5} n=2 n(p n)^{-4} .
\end{aligned}
$$

Thus, noting that $A$ and $A^{\prime}$ are $n$-by- $n$ and $n^{\prime}$-by- $n^{\prime}$ respectively, we have

$$
\left\|A^{\prime} \overline{\mathbf{1}^{\prime}}-p n \overline{\mathbf{1}^{\prime}}\right\|^{2} \frac{n^{\prime}}{n}-\|A \overline{\mathbf{1}}-p n \overline{\mathbf{1}}\|^{2} \leq 2(p n)^{-4} .
$$


This implies

$$
\begin{aligned}
& \left(1-\exp \left(-p n / c_{2}\right)\right)\left\|A^{\prime} \overline{\mathbf{1}^{\prime}}-p n \overline{\mathbf{1}^{\prime}}\right\|^{2}-\|A \overline{\mathbf{1}}-p n \overline{\mathbf{1}}\|^{2} \leq 2(p n)^{-4} \\
& \Longleftrightarrow\left\|A^{\prime} \overline{\mathbf{1}^{\prime}}-p n \overline{\overline{\mathbf{1}}^{\prime}}\right\|^{2}-\|A \overline{\mathbf{1}}-p n \overline{\mathbf{1}}\|^{2} /\left(1-\exp \left(-p n / c_{2}\right)\right) \\
& \quad \leq 2(p n)^{-4} /\left(1-\exp \left(-p n / c_{2}\right)\right) \\
& \Longleftrightarrow\left\|A^{\prime} \overline{\mathbf{1}^{\prime}}-p n \overline{\mathbf{1}^{\prime}}\right\|^{2}-\|A \overline{\mathbf{1}}-p n \overline{\mathbf{1}}\|^{2}\left(1+2 \exp \left(-p n / c_{2}\right)\right) \\
& \leq 2(p n)^{-4}\left(1+2 \exp \left(-p n / c_{2}\right)\right) \\
& \Longleftrightarrow\left\|A^{\prime} \overline{\mathbf{1}^{\prime}}-p n \overline{\mathbf{1}^{\prime}}\right\|^{2}-\|A \overline{\mathbf{1}}-p n \overline{\mathbf{1}}\|^{2} \\
& \leq 2(p n)^{-4}\left(1+2 \exp \left(-p n / c_{2}\right)\right)+2 \exp \left(-p n / c_{2}\right)\|A \overline{\mathbf{1}}-p n \overline{\mathbf{1}}\|^{2} \\
& \Longleftrightarrow\left\|A^{\prime} \overline{\mathbf{1}^{\prime}}-p n \overline{\mathbf{1}^{\prime}}\right\|^{2}-\|A \overline{\mathbf{1}}-p n \overline{\mathbf{1}}\|^{2} \\
& \leq 2(p n)^{-4}\left(1+2 \exp \left(-p n / c_{2}\right)\right)+2 \exp \left(-p n / c_{2}\right)(p n+o(1)) \\
& \Longleftrightarrow\left\|A^{\prime} \overline{\mathbf{1}^{\prime}}-p n \overline{\mathbf{1}^{\prime}}\right\|^{2}-\|A \overline{\mathbf{1}}-p n \overline{\mathbf{1}}\|^{2} \leq 3(p n)^{-4} \cdot
\end{aligned}
$$

Thus,

$$
||\left|A^{\prime} \overline{\mathbf{1}^{\prime}}-p n \overline{\mathbf{1}^{\prime}}\right||-\sqrt{p n}| \leq O\left((p n)^{-2}\right)+o(1)
$$

Next, using this, we derive

$$
\begin{aligned}
\left\|A^{\prime} \overline{\mathbf{1}^{\prime}}-p n^{\prime} \overline{\mathbf{1}^{\prime}}\right\|-\sqrt{p n} \mid & =\left\|\left(A^{\prime} \overline{\mathbf{1}^{\prime}}-p n \overline{\mathbf{1}^{\prime}}\right)+\left(p n \overline{\mathbf{1}^{\prime}}-p n^{\prime} \overline{\mathbf{1}^{\prime}}\right)\right\|-\sqrt{p n} \mid \\
& \leq\left\|A^{\prime} \overline{\mathbf{1}^{\prime}}-p n \overline{\mathbf{1}^{\prime}}\right\|-\sqrt{p n} \mid+\left\|p n \overline{\mathbf{1}^{\prime}}-p n^{\prime} \overline{\mathbf{1}^{\prime}}\right\| \\
& \leq O\left((p n)^{-2}\right)+\left(p n-p n^{\prime}\right)+o(1) \\
& =O\left((p n)^{-2}\right)+o(1) .
\end{aligned}
$$

Finally, the target bound is derivied by

$$
\begin{aligned}
||\left|A^{\prime} \overline{\mathbf{1}^{\prime}}-p n^{\prime} \overline{\mathbf{1}^{\prime}} \|-\sqrt{p n^{\prime}}\right| & \leq|| A^{\prime} \overline{\mathbf{1}^{\prime}}-p n^{\prime} \overline{\mathbf{1}^{\prime}}|-\sqrt{p n}|+\left|\sqrt{p n}-\sqrt{p n^{\prime}}\right| \\
& \leq O\left((p n)^{-2}\right)+\left|\sqrt{p n}-\sqrt{p n^{\prime}}\right|+o(1) \\
& \leq O\left((p n)^{-2}\right)+o(1)
\end{aligned}
$$


where the last iequality comes from

$$
\begin{aligned}
\sqrt{p n}-\sqrt{p n^{\prime}} \leq \alpha^{-2} & \Longleftrightarrow \sqrt{\alpha}-\sqrt{\alpha\left(1-\exp \left(-\alpha / c_{2}\right)\right)} \leq \alpha^{-2} \\
& \Longleftrightarrow 1-\left(1-\exp \left(-\alpha / c_{2}\right)\right)^{1 / 2} \leq \alpha^{-2-1 / 2} \\
& \Longleftrightarrow 1-\alpha^{-5 / 2} \leq\left(1-\exp \left(-\alpha / c_{2}\right)\right)^{1 / 2} \\
& \Longleftrightarrow\left(1-\alpha^{-5 / 2}\right)^{2} \leq 1-\exp \left(-\alpha / c_{2}\right) \\
& \Longleftrightarrow 1-2 \alpha^{-5 / 2}+\alpha^{-5} \leq 1-\exp \left(-\alpha / c_{2}\right) \\
& \Longleftrightarrow \exp \left(-\alpha / c_{2}\right) \leq 2 \alpha^{-5 / 2}-\alpha^{-5}
\end{aligned}
$$

since we let $\alpha=p n$.

TOMONORI ANDo CANON INC.

YOSHIYUKI KABASHIMA

Dept. of Comput. Intell. and Syst. Sci.

Tokyo Institute of Technology

E-mail address: kaba@sp.dis.titech.ac.jp

HisanaO TAKahashi

Dept. of Comput. Intell. and Syst. Sci.

Tokyo Institute of Technology

E-mail address: takahashi@sp.dis.titech.ac.jp

Osamu Watanabe

Dept. of Math. and Comp. Sci.

Tokyo Institute of Technology

E-mail address: watanabe@)is.titech.ac.jp

MaSAKI YAMAMOTO

Dept. of Mathematics

TOKAI UNIVERSITY

E-mail address: yamamoto@tokai-u.jp 